\title{
Reflets
}

Revue ontaroise d'intervention sociale et communautaire

\section{Être une immigrante noire africaine francophone à Toronto : vécu et perception des rapports de genre}

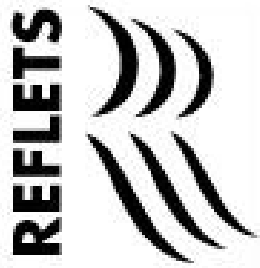

\section{Gertrude Mianda}

Volume 4, numéro 1, printemps 1998

Intervention en contextes minoritaires

URI : https://id.erudit.org/iderudit/026196ar

DOI : https://doi.org/10.7202/026196ar

Aller au sommaire du numéro

Éditeur(s)

Reflets : Revue ontaroise d'intervention sociale et communautaire

ISSN

1203-4576 (imprimé)

1712-8498 (numérique)

Découvrir la revue

Citer cet article

Mianda, G. (1998). Être une immigrante noire africaine francophone à Toronto : vécu et perception des rapports de genre. Reflets, 4(1), 34-52.

https://doi.org/10.7202/026196ar
Résumé de l'article

Cette étude qualitative traite du vécu et de la perception des rapports de genre des femmes immigrantes originaires de l'Afrique subsaharienne francophone à Toronto. Les contraintes de la vie d'immigrante engendrent une nouvelle situation qui les amène à vivre leurs rapports de genre dans une certaine tension. Leur perception de ces rapports en influencée par leur culture d'origine. Les Canadiennes, de leur côté, jugent les immigrantes africaines à partir des stéréotypesvéhiculés sur les Africaines. De là une certaine méfiance accentuée par les discriminations sexisteet raciste dont sont victimes les femmes immigrantes africaines au Canada. Aussi se sentent-ellesbeaucoup plus solidaires des hommes de leur communauté qui vivent la même exclusion.
Tous droits réservés $(\subset$ Reflets : Revue ontaroise d'intervention sociale et communautaire, 1998
Ce document est protégé par la loi sur le droit d'auteur. L'utilisation des services d’Érudit (y compris la reproduction) est assujettie à sa politique d'utilisation que vous pouvez consulter en ligne.

https://apropos.erudit.org/fr/usagers/politique-dutilisation/ 


\section{Être une immigrante noire africaine franco- phone à Toronto :}

\section{Vécu et perception des rapports de genre $^{1}$}

Cette étude qualitative traite du vécu et de la perception des rapports de genre des femmes immigrantes originaires de l'Afrique subsaharienne francophone à Toronto. Les contraintes de la vie d'immigrante engendrent une nouvelle situation qui les amène à vivre leurs rapports de genre dans une certaine tension. Leur perception de ces rapports en influencée par leur culture d'origine. Les Canadiennes, de leur côté, jugent les immigrantes africaines à partir des stéréotypes véhiculés sur les Africaines. De là une certaine méfiance accentuée par les discriminations sexiste et raciste dont sont victimes les femmes immigrantes africaines au Canada. Aussi se sentent-elles beaucoup plus solidaires des hommes de leur communauté qui vivent la même exclusion.

\section{Gertrude Mianda}

Professeure, Sociologie/Études des femmes, Collège Glendon, Université York

La nouvelle immigration au Canada se caractérise de manière générale par l'arrivée de personnes originaires des pays du Tiersmonde avec la présence notamment de plus en plus de femmes (Labelle 1990 :70). En 1996, 55,38 \% d'immigrants provenaient de l'Asie et du Pacifique, 16,11 \% de l'Afrique et du MoyenOrient, 8,21 \% de l'Amérique du Sud et Centrale contre 17,72\% d'Europe et du Royaume-Uni et 2,58 \% des États-Unis (Immigration Canada 1997). Dans la mesure où la migration définitive s'accompagne du désir de conserver son identité ethnique (Berry 1976:8), la diversité culturelle au Canada, qui se 
proclame d'ailleurs un pays multiculturel, est par conséquent un fait incontestable. Cependant, comme le constatent certains chercheurs, le fait de garder des traits culturels des pays d'origine différents des modèles de la culture dominante paraît constituer une entrave à l'intégration des nouveaux arrivants. Il provoque des réactions d'intolérance, de rejet de la part de la population de souche (Langlois, Laplante et Levy 1990:18). Dans le même sens, un sondage Gallup de janvier 1992 indique la crainte des Canadiens face à l'immigration croissante qui menacerait la culture nationale blanche.Ainsi, un Canadien sur trois refuse l'immigration afin de préserver la culture nationale blanche, mais également du fait que les immigrants ne semblent pas respecter certains principes de base de la société canadienne comme la séparation de l'Église et de l'État et l'égalité des sexes (Helly 1996:66). Ces immigrants considérés comme de culture différente proviennent généralement des pays du Tiers-monde et présentent plus de difficulté d'intégration que les immigrants d'origine européenne (Rogel 1989:9).

Compte tenu de cette attitude à l'égard des immigrants, je traite, dans cette étude exploratoire, du vécu et de la perception des rapports de sexe par les Africaines noires immigrantes francophones dans le contexte canadien et particulièrement celui de Toronto. Il existe une abondante littérature sur les immigrantes au Canada, comme le démontre le bilan exhaustif qu'en fait $\mathrm{M}$. Labelle (1990). Ces études portent globalement sur l'intégration fonctionnelle des femmes de multiples communautés ethniques avec la diversité de problèmes qu'elle soulève, à savoir la discrimination sexiste, raciale ainsi que leur inscription ou non, de manière voilée ou évidente, dans les politiques d'immigration et dans les institutions. Cependant, en vertu de leur faible représentation démographique et d'une histoire d'immigration encore récente, les Africaines noires, de par leur vécu, ne semblent pas intéresser suffisamment les chercheuses. Depuis celle de Kusozi (1988), une des plus récentes études sur les femmes africaines de Toronto (Nakanyike, Musisi, Turrittin 1995) traite principalement de leur intégration économique. Elle met également l'accent sur les discriminations de sexe, de race dont sont victimes les Africaines. 
Elle aborde de façon relative les relations entre les sexes telles que les vivent les Africaines dans le contexte canadien. De ce point de vue, cette étude s'inscrit dans le prolongement des travaux antérieurs sur les immigrantes en général.

Tout en traitant également de ces questions, ma recherche se concentre spécifiquement sur les rapports de sexe à partir de l'expérience des Africaines et leur perception dans le pays d'accueil. Pour ce faire, j'ai réalisé des entrevues semi-structurées avec des Africaines immigrantes vivant à Toronto et à Montréal ${ }^{1}$, sur le modèle biographique (Bertaux 1980). Dix de ces entretiens, ceux de résidantes de Toronto, constituent le corpus de l'analyse que je tente d'exposer dans cet article. Ces Africaines se sont toutes mariées ${ }^{2}$ dans leur pays d'origine où elles avaient vécu en couple et la majorité d'entre elles vit au Canada depuis plus de 10 ans. Elles proviennent de divers pays d'Afrique noire francophone et sont soit chrétiennes soit musulmanes. Cette démarche m'a paru adéquate pour ce type de recherche dans lequel mes interlocutrices devaient parler de leur expérience quotidienne autour des rapports de sexe. Moi-même immigrante d'origine africaine,j'ai été parfois amenée à partager par des signes d'approbation évidente les dires de mes interlocutrices. Certaines féministes, à l'instar d'Oakley (1981), Ramazanoglu (1989), Devault (1990) et Cotterill (1992), soulignent l'importance de l'interaction entre la chercheuse et la répondante. Sans occulter le fait, que rappelle si bien Ramazanoglu (1986), de la multiplicité d'expériences des femmes selon leur classe, leur race, leur ethnicité, mon expérience de femme immigrante africaine peut en certains points différer de celle de mes répondantes. Ce faisant, mon attitude fut surtout celle de l'écoute attentive.

D'autre part, les rapports sociaux de sexe étant des relations complexes qui varient dans l'espace et le temps, selon les situations et le contexte socio-économique, politique et culturel, certains enjeux sont apparus plus importants que d'autres pour mes interlocutrices dans leurs rapports avec les hommes époux dans l'environnement canadien. L'autorité du mari, le partage de la charge domestique, la prise de décision au sein du ménage, sont revenus plus couramment dans les propos des Africaines que, par 
exemple, la gestion du revenu ou l'exercice d'une profession par l'épouse.Ainsi,l'égalité des sexes dans ce cas pourrait se comprendre comme une égalité autour de ces facteurs. C'est donc sur la base de ceux-ci que s'articule l'analyse que j'essaie d'esquisser.

Un autre aspect, non moins secondaire, est d'une part, la perception que les Canadiennes de souche ont des Africaines par rapport à leur relation avec les hommes et d'autre part, la perception que les Africaines se font des relations des Canadiennes avec les hommes. La connaissance de ces points de vue des Africaines est importante, surtout dans un milieu multiculturel comme Toronto où la personne intervenante sociale est appelée à oeuvrer aussi souvent avec des immigrantes des différentes cultures. Elle facilite non seulement l'échange interculturel mais peut aussi permettre à l'intervenante de comprendre l'adhésion ou la résistance des Africaines à certaines valeurs de l'égalité des sexes telle que comprise dans la société canadienne. En effet, en plus de certaines attitudes et compétences, l'intervention en milieu culturel commande la connaissance de diverses cultures (Cohen 1993; Bellfort 1993).

Pour une meilleure compréhension du vécu des Africaines immigrantes sur leurs rapports sociaux de sexe, il convient de retracer d'abord les étapes de leur établissement définitif au Canada, en l'occurence à Toronto. Cela permettra de saisir, dans un deuxième volet, la manière dont elles vivent les relations avec leurs conjoints dans le contexte canadien et enfin d'éclairer le regard qu'elles posent sur les valeurs d'ici quant aux rapports de sexe $e^{3}$.

\section{Des Africaines immigrantes de Toronto et leur itinéraire : Immigrer pour perdre un statut socio- économique décent}

Le Québec a été la porte d'entrée au Canada pour cinq de mes dix interlocutrices. En effet, elles ont rejoint leur époux ${ }^{4}$ qui les 
avait précédées pour raisons d'études. L'arrivée au Québec s'inscrit ainsi dans la perspective de décrocher un diplôme de niveau supérieur, particulièrement pour le conjoint, afin d'accéder à une position socio-économique convenable dans le pays d'origine. Car, en dépit de l'ascension politique à caractère arbitraire et souvent ethnique, la possession d'un diplôme en Afrique, notamment de niveau universitaire, garantissait encore, il n'y a pas si longtemps, une position socio-économique prestigieuse. Cependant, l'instabilité politique persistante et la détérioration constante des conditions matérielles d'existence ont contraint les étudiants africains et leurs familles à demeurer au Canada comme immigrants. Ils se retrouvent alors, à plus d'un égard, dans la même situation que l'immigrant classique. Celui-ci est attiré par l'Occident dans le courant inverse de l'internationalisation du capital, comme le soulignent les études raciales (Lavigne 1987:29_ 42), pour vendre sa force de travail en espérant améliorer sa situation. Même si au-delà de l'internationalisation du capital, le phénomène d'immigration internationale tend à s'expliquer par l'image idyllique que le Nord projette vers le Sud, entre autres, à travers les médias (SIN 1993). N'empêche que l'immigrant est très vite désillusionné. Les Africaines expérimentent cette dure réalité, surtout par leurs conjoints qui, malgré le diplôme obtenu au Canada, attendent des années un emploi correspondant à leur formation. Faute de mieux, ils finissent souvent par accepter des emplois subalternes pour survivre dignement. La situation est encore plus dramatique pour ceux qui sont entrés comme réfugiés ou ceux qui n'ont pas eu leur diplôme au Canada. Pour ceux qui sont arrivés au Canada par le Québec, le quitter s'avère une voie prometteuse.

Mon mari avait perdu un contrat qu'il avait à Montréal parce qu'il ne parlait pas l'anglais. Comme il ne trouvait pas du travail, il a préféré qu'on démenage pour Toronto. Au moins on peut apprendre l'anglais. Qui sait si un jour.

Nous avons quitté Montréal parce que mon mari le voulait. Il n'y a pas beaucoup de travail, donc mon 
mari a décidé qu'on devait venir à Toronto pour trouver du travail. Il a eu de bons contacts et au bout de deux semaines, il a trouvé un petit boulot. Ce n'est pas ce qu'il y a de mieux mais c'est mieux que rien.

Pour ces Africaines, la vie à Toronto ou à Montréal n'offre pas des perspectives valorisantes par rapport à la position économique qui leur conférait un certain statut social de prestige dans leur pays d'origine. À défaut de mener une carrière, elles avaient une source de revenu autonome, soit un petit commerce, une petite entreprise de couture ou de coiffure. Par ce fait, ces Africaines considèrent qu'elles avaient là un statut socio-économique respectable leur assurant une certaine dignité.

En venant ici, j'ai perdu un statut. Je travaillais. Ce que je gagne ici, c'est le cadre que je peux offrir à mes enfants pour les études. Encore là, il faut parfois s'interroger: qu'est-ce que je perds, ces enfants là n'auront jamais l'éducation que j'aurais voulu leur donner. Ici là, il faut même craindre ses propres enfants. Ici, je ne suis plus secrétaire mais j'ai dû travailler dans des manufactures, mentalement c'est très dur. J'ai sacrifié mon statut pour le bien de mes enfants.

Malgré le niveau d'étude qui leur permet l'accès à un emploi correspondant plus ou moins à leurs qualifications dans leur pays d'origine, les Africaines ne dénichent pas facilement un emploi. Il faut passer par un long processus d'adaptation en acceptant, pour survivre, le premier travail disponible, en vue d'avoir l'expérience canadienne exigée. D'autre part, la reprise d'un autre cycle d'études semble un moyen de parfaire la formation dans l'espoir d'intégrer le marché du travail. Mais le fait d'être femme et en plus noire se présente comme un obstacle qu'il faut quotidiennement surmonter.

Dans mon travail, on croit que parce que je suis une femme noire africaine, donc, je ne connais pas. Je ne connais rien. Le cas typique, quand les gens téléphonent, ils ne pensent jamais que je suis une fermme 
noire, jamais, jamais. Et puis bon, ils sont plus ou moins satisfaits de la première entrevue. Mais quand ils arrivent, ils sont surpris. Et puis après je vois un changement dans leur attitude. L'appréciation vient avec le temps, avec la découverte de la personne. Mais à priori, quand ils voient qu'il s'agit d'une noire, c'est négatif. Après, ils se rendent compte que je fais mon boulot, je suis polie, je suis correcte et efficace, et petit à petit l'appréciation vient. A priori, tu n'es rien, tu dois faire tes preuves et tu dois faire plus que les autres.

(...) En fait, j'ai deux patrons: une femme et un homme. Du côté de la dame, elle a plus d'expérience avec les gens, elle a plus de respect pour les autres. Cependant, du côté du monsieur, il a essayé de prendre contrôle sur moi. Peut-être parce que c'est un homme. Mais je ne me suis pas laissé faire. Et aujourd'hui, j'ai gagné du respect. Mais j'ai dû me battre pour cela. Moi je ne me fais pas de complexe. Je ne tolère pas que quelqu'un croit que je suis moins bonne parce que je suis noire, je suis Africaine.

Ces Africaines se sentent victimes de discrimination raciale. L'intégration dans le milieu de travail se révèle un combat perpétuel. Un éternel recommencement quotidien jusqu'à ce qu'elles puissent convaincre par la qualité de leur travail. Parce que femmes noires, elles font face à une remise en question constante de leur compétence. C'est un facteur de stress supplémentaire dans une conjoncture d'emploi déjà précaire qui fait accentuer le sentiment d'humiliation. Être obligées de travailler durement dans des emplois les moins bien rémunérés, même lorsqu'on a des qualifications suffisantes, semble être le lot des Africaines immigrantes. Elles le vivent comme une perte de leur statut en même temps qu'elles doivent assumer des responsabilités domestiques plus lourdes dans un contexte où elles ne bénéficient plus d'un support familial quelconque. 


\section{Une conjugalité dans la tension}

En fait, pour les Africaines, la vie d'immigrante loin du contexte africain permet le développement des relations de couple plus intenses, une nouvelle forme de conjugalité. La composition familiale est atomisée. Elle se réduit très souvent aux conjoints seuls et à leurs enfants. En somme il s'agit d'une famille nucléaire. De manière générale, ces Africaines immigrantes connaissent alors la naissance d'une conjugalité beaucoup plus intime que celle vécue en Afrique. Si celle-ci permet parfois de resserrer de plus en plus le lien affectif dans le couple, elle peut aussi produire une certaine tension. En effet, en Afrique, malgré d'autres occupations, une profession, un commerce, ces femmes pouvaient organiser assez aisément leur temps. Le fait de vivre toujours entouré d'autres membres de la famille empêchait une conjugalité très intime certes, mais permettait le partage de certaines charges domestiques par des femmes de la parenté. Cependant, l'expérience d'être une immigrante noire dans ce contexte est non seulement marquée par la discrimination, l'humiliation, une certaine perte de dignité mais elle alourdit considérablement la charge domestique pour les Africaines. Dans la mesure où culturellement les obligations domestiques incombent aux femmes, le manque d'aide fait qu'elles sont souvent confrontées à une surcharge de travail. L'organisation d'une journée, pour celles qui travaillent à l'extérieur, est une course à la montre sur un mode différent de ce qu'elles ont connu en Afrique.

Le matin, je dépose les enfants à la garderie. On quitte la maison à $7 \mathrm{~h} 15$. On attend devant la première garderie que les portes s'ouvrent. À $7 \mathrm{~h} 25$, on entre. Mais je dois attendre à 7 h 30 parce qu'il n'y a pas d'assurance au cas où quelque chose arriverait. Donc, je dépose d'abord le bébé après je dépose la grande. A chaque fois, il faut entrer et signer. C'est des «in and out». Je quitte l'école de la grande, ensuite, je vais travailler et j'arrive souvent vers 8 h ou 8 h 05. Après 
le boulot, je dois m'occuper du ménage. Il faut apprêter le repas. Mais, je m'organise car je fais la plupart des repas le week-end. Je conserve dans des bols en plastique. J'ai toujours fait mes affaires toute seule. Mon mari ne s'est jamais occupé des travaux domestiques. Il n'y a pas de partage des tâches. Soit mon mari n'est pas là ou s'il est là, il doit se reposer. La seule chose qu'il accepte de faire, c'est le ménage le samedi.

Mon mari ne fait pas les travaux ménagers. Ce n'est pas son rôle. L'aide que nous trouvons chez nous n'existe pas ici. Les femmes ici demandent que les hommes participent aux travaux domestiques, mais tu sais le mariage c'est comme une entreprise. Dans un ménage chaque personne a son rôle. Dans toute entreprise il y a un chef d'entreprise. Le chef, le capitaine, c'est l'homme. La femme, c'est la gardienne de valeurs. C'est elle qui fait exécuter les choses. Je ne peux pas lui demander de faire le travail de la maison. S'il veut aider ça c'est différent. Ici, le contexte est différent. Il peut le faire s'il veut bien. Mais ce n'est pas son rôle. C'est sûr que c'est très dur pour moi d'être partagée comme cela à courir après le temps, mais je sais m'en sortir.

Il y a celles qui acceptent d'assumer seules, au nom du respect des rôles sexués, toutes les responsabilités domestiques, en dépit des conditions sociales différentes de celles de l'Afrique. Elles s'accommodent de la double charge de travail afin de préserver les valeurs culturelles, faisant lecture fonctionnaliste du rôle des sexes et acceptant l'autorité du conjoint libéré des travaux ménagers. Par contre, les autres ressentent comme un lourd fardeau le fait de s'occuper seules de toute la charge domestique en plus de travailler à l'extérieur. Ces dernières ne sont pas à l'abri de certains conflits conjugaux et sont parfois victimes de médisance de la part de leurs amis africains ou de compatriotes du même pays d'origine. En Afrique, la conjugalité englobait la parenté. Essentiellement, la femme ajustait son comportement par rapport à cette dernière. Au Canada, le réseau de relations entre Africains 
se substitue à la parenté et joue sensiblement le même rôle que celle-ci. Ainsi, le dénigrement des amis peut parfois avoir une incidence sur le comportement du couple notamment en public, amenant les conjoints à se conformer en apparence aux normes culturelles de conduite entre époux. Celles dont le conjoint participe quelque peu aux travaux domestiques manipulent le jeu de l'apparence afin de préserver le statut social reconnu à l'époux dans la communauté.

Quand on est mariée, il faut faire des sacrifices. Ce qu'une femme est capable de supporter ou d'accepter, un homme n'est pas capable de le faire. Et moi, j'ai décidé que je ne pouvais plus supporter le fait par exemple d'être mariée et d'avoir la responsabilité des enfants toute seule. J'ai trouvé que cela ne valait pas la peine de prétendre être mariée si c'est pour faire tout, toute seule. Cela n'a pas de sens si c'est juste pour paraître. Alors, je ne sais pas à qui je fais plaisir en paraissant. En tout cas, pas à moi.

En Afrique tout aurait été différent parce que le contexte émotionnel et social aurait été différent. J'aurais été entourée d'une famille. J'aurais eu un support affectif qui aurait permis d'équilibrer le manque de présence de mon mari. Je n'aurais pas souffert de la même manière. J'aurais minimisé par exemple le fait que mon mari ne soit pas impliqué dans les tâches ménagères. J'aurais eu une autre source de soutien affectif malgré son absence, $j$ 'aurais eu une source de soutien pratique, par exemple, pour les enfants. J'aurais eu de l'aide. J'aurais eu un "nounou». J'aurais eu quelqu'un pour faire le ménage pour moi, soit pour faire la cuisine. Tout ce qui pèse sur mes épaules aujourd'hui, je n'aurais pas eu ça. J'aurais eu certainement d'autres problèmes mais sur le plan de l'organisation pratique de tous les jours, je n'aurais pas eu ça. 
Le partage des travaux domestiques se fait dans le tiraillement. Il s'opère, pour certaines, au prix d'une demande persistante, non pas en remettant nécessairement en question l'autorité du mari ni son rôle. Simplement parce que confrontées à une surcharge de travail et ne pouvant plus profiter de l'aide qu'elles recevaient en Afrique, elles se trouvent dans l'obligation de solliciter l'apport du conjoint surtout lorsqu'il n'y a pas de grands enfants dans la famille. Cependant pour d'autres, il y a une contestation effective de l'ordre établi dans le mariage. Dans ce cas, cela se produit assez souvent dans la tension ou crée une situation de conflit latent.

Une femme africaine croit au mariage et au fait de rester mariée quoi qu'il advienne. Je le vois autour de moi. Il $\gamma$ a beaucoup d'Africaines qui sont convaincues qu'être malheureuse fait partie intégrante du mariage. Que la tristesse, le malheur, prendre toute les responsabilités domestiques, c'est ça réussir son mariage, même si cela est trop lourd. Etre mariée est important pour une femme,je te parle de femmes africaines qui vivent ici au Canada, pas en Afrique. Le problème est différent en Afrique parce que l'apport des autres membres de la famille est là. On ne ressent pas les reponsabilités de la même façon et ça ne dérange donc pas de rester mariée malgré tout. Ce que je trouve difficile ici, c'est le stress qu'on est obligée de vivre en plus de supporter un mari qui ne vous aide pas et qui se réserve le droit de vous tabasser. Je trouve cela intolérable. Tu vois ce que je trouve difficile, c'est d'avoir tellement de choses à faire et d'être obligée de le faire seule alors que je suis mariée. Alors je me dis, je n'ai pas besoin d'un mari qui n'aide pas et qui ajoute aux problèmes. Non,j'ai décidé que je n'avais pas besoin de plus de stress. Moi,j'ai arrêté de supporter, j'ai arrêté de faire des efforts. Là, alors, c'est la dégringolade. J'ai changé parce que j'ai estimé que je méritais mieux que ça.

La perte du soutien familial pour les travaux domestiques ainsi que le vécu canadien façonnent la relation à l'égard du mari. 
Pour certaines, ce changement occasionne le réaménagement du partage relatif du travail domestique. Pour d'autres, il donne lieu à une contestation, une rupture d'avec la vision du mariage. Cependant, il n'y pas une adhésion totale à l'idée d'égalité de sexe dans la division des tâches domestiques; ni à ce qui est perçu comme étant la conception canadienne du mariage, tel que le suggère le propos suivant.

Ici la conception du mariage est différente. Le sexe ne va pas seulement avec le mariage. Déjà, ici même, on peut remarquer qu'au Québec et à Toronto, c'est très différent. Au Québec, les femmes sont très indépendantes. Elles sont trop féministes. Quelques fois, il y a même un peu d'exagération. Mais les femmes ont lutté quand même pour acquérir leurs droits. Ce que j'ai perçu au Québec, c'est qu'on peut vivre sa sexualité de manière épanouie sans pour autant être obligée de se marier. En Ontario, je n'ai pas remarqué la même liberté sexuelle que j'ai remarquée an Québec. Aussi peut-être parce qu'en Ontario, on a l'impression que tout le monde vient de partout sauf d'ici.Vu qu'il y a trop d'immigrants, il $y$ a un apport culturel important, on ne perçoit pas des "pure laine».

L'idée de séparer la procréation du mariage ne semble pas être exprimée avec clarté dans ce propos mais elle me parait suggérée dans la phrase suivante:«vivre sa sexualité de manière épanouie sans pour autant être obligée de se marier». Ceci indique une certaine distance par rapport à la conception africaine du mariage, lequel est inconcevable sans progéniture (Mbite 1972:143; Kenyatta 1967:116). Paradoxalement, rien n'indique qu' on puisse conclure qu'il y a acceptation totale du modèle des relations de genre de la société canadienne. Par ailleurs, il ressort également de ce propos une reconnaissance du combat mené par les femmes canadiennes de souche, signifiant par là, dans une certaine mesure, le rapprochement des immigrantes africaines des revendications des Canadiennes d'origine. 


\section{D'ici mais toujours d'ailleurs : Solidarité féminine versus discrimination}

Les immigrantes d'origine africaine sont confrontées à la double charge de travail, comme les autres femmes du Canada, malgré une plus grande implication du conjoint dans certaines tâches. Cette situation commune ne suffit pas à constituer une source potentielle de solidarité comme le révèlent le discours de ces Africaines.

Les femmes d'ici ne sont pas solidaires avec les femmes africaines. On a beau être une fermme, mais il y a la barrière de la peau. Une femme blanche, d'une façon ou d'une autre, se croira toujours supérieure à toi. Il n'y a rien à dire. Il n'y a pas de vraie solidarité, même si elles vivent parfois les mêmes problèmes que nous. Par exemple, si j'observe autour de moi mes amies blanches ontariennes d'ici, il n'y a pas non plus autant d'aide à la maison que ça. Cela dépend, l'homme fera ce qu'il a envie de faire. Il ne se sentira pas la même responsabilité que la femme. Il va par exemple déneiger peut-être parce que lui, ça ne le dérange pas de faire cela. Par contre, il ne fera pas toujours nécessairement la vaisselle. Dans ce sens là, il $y$ a des maris d'ici qui ne font rien. Et à l'opposé, on peut trouver également des hommes africains qui font tout pour leur épouse. Malgré cela, les femmes d'ici nous jugent toujours comme des femmes soumises, exploitées. La femme qui retombe toujours dans les mêmes erreurs d'accepter tout de l'homme, une femme trop bonne qui va faire l'esclave de l'homme. Il y a toujours ce cliché qui est là.

Moi, je n'aime pas discuter avec les femmes d'ici; je ne discute pas avec les féministes. Je n'argumente même plus. Cela ne vaut pas la peine. Elles ont leur conception. Pour elles, nous sommes des femmes africaines donc des femmes exploitées, soumises. Tu vois même dans le 
cours de langue pour apprendre l'anglais, la dame qui nous donnait le cours tenait toujours ce langage là. Les femmes Africaines font ceci, elles font cela. Il faut laisser participer les hommes au travail à la maison, changer les couches $d u$ bébé, faire la vaisselle. En fait, il $y$ a des choses que moi,je trouve normales et qu'elle ne peut pas comprendre. C'est normal que mon mari prenne des décisions, qu'il gère l'argent. J'ai été élevée comme ça,je n'ai pas vu mes grandes soeurs exiger de leur mari de faire la vaisselle.Vois-tu, avec tout le racisme qu'on connaît ici, mon mari déjà ne se trouve pas du travail facilement. Il se sent humilié et si en plus je dois lui imposer de faire encore les travaux à la maison, tu vois ce que ça donne. C'est pourquoi mes amies sont Africaines, je ne me tiens pas avec les femmes d'ici.

En se basant, entre autres, sur la presque absence d'implication des maris africains dans les tâches du ménage, les Canadiennes de souche semblent poser un regard condescendant sur les immigrantes africaines, les forçant à exprimer avant tout leur identité africaine au lieu de proclamer une forme de sororité avec les femmes d'ici. Il s'agit vraisemblablement d'une attitude défensive. Car loin de nier tout simplement les inégalités de sexe propres à leur culture, les immigrantes africaines en sont plutôt bien conscientes. Elles le disent.

Ici on ne lie pas la vie d'une femme au mariage alors qu'en Afrique, on la lie. Tu as plus de considération si tu es mariée. Même ici dans le milieu afro-canadien, quand tu n'es pas mariée, on ne te respecte pas.

Une femme doit être soumise. Elle doit se marier pour vivre avec un homme. Elle doit être présente pour son mari. Elle doit assumer sa responsabilité à l'intérieur du ménage. Une fille célibataire, c'est mal perçu parce qu'elle n'est pas mariée. En Afrique, l'identité féminine doit se définir par le mariage. Ici, on est femme parce qu'on est un être humain. Il faut reconnaître que les 
femmes d'ici ont lutté pour ça, elles se sont battues. Surce plan, je les envie même si je ne suis pas toujours d'accord avec elles sur certaines choses.

Le discours des Africaines sur le statut de la femme est paradoxal. D'un côté, elles présentent la soumission de la femme comme un fait de la culture. D'autre part, elles sont conscientes de l'inégalité des rapports de sexe tout en n'osant pas ouvertement la contester. Dans la mesure où elles reconnaissent les mérites des femmes blanches d'avoir gagné leur place dans la société au même titre que les hommes, elles admettent l'inconfort de leur situation. L'attachement culturel semble agir, pour certaines, comme une force normative puissante, au point de neutraliser toute contestation possible.

Par ailleurs, la relation avec les femmes blanches demeure encore entachée de l'empreinte de l'histoire coloniale. Elle traduit un rapport de pouvoir entre femmes qui s'inscrit dans la tradition coloniale. Le regard hautain que les Africaines perçoivent de la part des femmes blanches s'inscrit dans cette logique. Ainsi, l'attitude de ces dernières vis-à-vis des immigrantes africaines semble s'approcher du racisme au lieu d'exprimer la solidarité qui sans doute sous-tend leur jugement sur les Africaines.

D'autre part, les rapports entre les immigrantes d'origine africaine et les femmes noires originaires des Caraibes sont caractérisés également, selon les premières, par une certaine forme de mépris de la part des secondes.

Les femmes noires des îles socialisent facilement avec les femmes blanches. Elles imitent facilement les blanches et nous considèrent aussi comme des femmes soumises. Nous n'avons de commun que la couleur de la peau.

On n'est pas pareilles avec les femmes noires des îles. Culturellement, on est très différentes. Je suis allée un peu dans des groupes afro-canadiens. J'ai arrêté parce que je considère qu'on n'a pas les mêmes problèmes. On n'est pas pareilles. Dans cette société, je veux dire au Canada, il y a des noires des îles, des noires 
d'Afrique, des blancs, des indiens, etc.... Ce qui me frappe, c'est que les communautés ne se mélangent pas. On se côtoie sans jamais se mélanger. Une Jamaïcaine, quand elle parle d'une fermme africaine, c'est comme si elle est dégoutée. Pour elle, la femme africaine aime le mariage. La plupart des femmes jamaïcaines que je connais ne sont pas mariées. Elles ne comprennent pas comment les femmes africaines sont là à traîner avec les hommes qui parfois les font souffrir. Elle nous considèrent également comme des femmes soumises.

Les différences culturelles à propos des relations de genre semblent nuire au développement de solidarité entre les femmes immigrantes noires d'origine africaine et les blanches ainsi que les femmes noires d'autres cultures. Dans ce contexte, la sororité ne parait pas transcender les barrières raciales et culturelles. Une question se pose de toute évidence: est-ce que la solidarité féminine pourait être assez forte pour parvenir à anéantir la persistance d'un racisme particulier envers les Noirs dans le monde? Être Africain, soutient T. Pujolle (1994:30), c'est devoir, indéfiniment, affirmer sa dignité face à l'autre. Les Africaines immigrantes expérimentent cette réalité aussi bien dans leur milieu de travail que dans la vie courante, comme il ressort de leurs propos.

\section{Être francophone à Toronto}

Le Canada est connu comme un pays bilingue. Pourtant, la personne immigrante qui maitrise une de deux langues officielles, en particulier le français, court le risque d'être confrontée au problème d'intégration linguistique, notamment à Toronto. Cela constitue un handicap de plus pour réussir son intégration économique.

Les francophones à Toronto, on est une minorité. C'est difficile d'être francophone ici, dans le sens où il y a 
une ségrégation contre les francophones. Les gens ont aussi peur de la séparation. Si on n'apprend pas l'anglais, c'est très difficile de se trouver du travail. Si tu ne parles pas très bien l'anglais, ou avec beaucoup de fautes, l'employeur te dit souvent: "The customers won't be happy". C'est pas évident non plus que si on parle anglais, on est accepté facilement. Les gens me disent souvent: "You have a funny accent, a french accent». Donc, mon accent va rester toujours pour m'identifier comme francophone d'origine africaine parce qu'il y a aussi cet accent là et je ne cherche pas non plus à le perdre.

Métropole du Canada, reconnue comme un des poumons économiques du pays, Toronto se présente comme la ville de l'espoir d'un statut socio-économique plus ou moins digne. À défaut de cela, elle offre l'opportunité d'apprendre l'anglais qui, pour ces immigrantes francophones, semble être la langue indispensable pour percer le marché du travail.Ainsi, le bilinguisme n'est efficace que sur papier, dans les documents officiels et dans la vie courante, surtout dans une région où le français est minoritaire.

\section{Conclusion}

Cette étude s'inscrit dans le cadre d'une recherche plus large. Dans ce sens, elle pose le jalon d'une analyse encore à approfondir. J'ai exploré essentiellement quelques éléments qui reviennent dans les propos des femmes. À travers ceux-ci, elles disent leur expérience d'immigrantes noires d'Afrique. Ce facteur de la race me parait capital dans la compréhension de la situation des immigrantes originaires de l'Afrique subsaharienne autant sur le marché du travail, où le racisme omniprésent ressort avec acuité quand il s'agit d'une femme, que dans la vie quotidienne. En outre, il apporte, avec la dimension culturelle, un éclairage sur la vision que les Africaines immigrantes ont des rapports de sexe et 
sur leur vécu dans la société canadienne où elles connaissent une forme de conjugalité nouvelle mais problématique. Le fait d'être noire, combiné aux stéréotypes associés aux femmes africaines à partir desquels les femmes blanches canadiennes posent un regard qui parait exprimer une certaine forme d'arrogance plutôt que de la compassion, tend à saper les bases d'une réelle solidarité féminine. Il en découle que les immigrantes africaines se solidarisent facilement entre elles et avec les hommes de la communauté qui comme elles, sont victimes de la même discrimination raciale.

\section{Bibliographie}

BERRY, John W, Rudolf KALN, Donald M.TAYLOR (1976). Attitudes à l'égard du multiculturalisme et des groupes ethniques au Canada.

BERTAUX, D. (1980). «L'approche biographique: sa validité méthodologique, sa potentialité», Cahier internationaux de sociologie, vol.69, 197-225.

BELLFORT, S. (1993). «Au carrefour de plusieurs vérités: une réflexion sur la pratique en milieu scolaire pluriculturel», Intervention, vol. 96, 36-46.

COHEN, Émerique (1993). «L'approche interculturelle dans le processus d'aide», Revue française de Service Social, vol.171, 7-19.

COTTERILL, Pamela (1992). «Interviewing Women. Issues of Friendship,Vulnerability and Power», Women's Studies International Forum, vol. 5, no 6, 29-42.

DEVAULT, Marjorie L. (1990). «Talking and Listening from Women’s Standpoint: Feminist Strategies for Interviewing and Analysis», Social Problems, vol. 37, no 1, 96-113.

HELLY, Denise (1996). Le Québec face à la pluralité culturelle 1977-1984. Un bilan documentaire des politiques, Québec, Les Presses de l’Université Laval.

IMMIGRATION CANADA (1997). Faits et chiffres 1996. Aperçu de l'immigration, Ottawa, Citoyenneté et Immigration Canada.

KASOZI,A.B.K (1988). The Integration of Black African Immigrants in Canadian Society: A Case Study of Toronto, CMA, 1986. Toronto, Canadian African Neewcomer Aid Center of Toronto (CANACT).

KENYATTA J. (1967). Au pied du mont Kenya, Paris, Maspéro.

LABELLE, M. (1990). «Femmes et immigration au Canada: Bilan et perspective», Études ethniques au Canada, vol.22, no 1, 67-82.

LANGLOIS, Jacques, Pierre LAPLANTE et Joseph LEVY (1990). Le Québec de demain et les communautés culturelles, Montréal, Éditions du Méridien.

LAVIGNE, Gilles (1987). Les ethniques et la ville, Montréal, les Editions du Préambule, Collection Science et théorie.

MBITI J. (1972). Religion et philosophie africaine, Yaoundé, Editions Clé. 
NAKANYIKE B, Musisi, Jane TURRITTIN (1995). African Women and the Metropolitan Toronto Labour Market in the 1990s. Migrating to A Multicultural Society in A Recession, Toronto.

OAKLEY, An (1981). «Interviewing Women: A Contradiction in Terms», in Helen Roberts (Ed.), Doing Feminist Research, London, Routledge and Kegan Paul, 30-61.

PUJOLLE, Thérèse (1994). L'Afrique noire, France, Dominos/Flammarion.

ROGEL, Jean -Pierre (1989). Le défi de l'immigration, Québec, Institut québecois de recherche sur la culture.

RAMAZANOGLU, Caroline (1986). Feminism and the Contradictions of Oppression, London, Routledge.

RAMAZANOGLU, Caroline (1989). «Improving on Sociology :The Problems of Taking a Feminist Standpoint», Sociology, vol.23, no3, 427-442.

SIN, Naï (1993). «Mondialisation et migrations : l'axe Sud-Nord», Les migrations internationales, Lausane, Payot.

\section{Notes}

1. J'utilise l'expression "genre» comme traduction francaise de gender qui exprime le fait de la construction sociale de la différence entre les sexes. La littérature francophone préfere plutôt le terme rapports sociaux de sexe.

2. Le fait d'être mariée dans le pays d'origine est important car il permet à mes interlocutrices d'établir une comparaison entre leur situation là-bas et celle vécue ici quant aux rapports de sexe.

3. On ne peut nier ici le fait que ce vécu, tout adapté soit-il à la vie au Canada, est influencé par la représentation des rapports de genre de la société d'origine.

4. Malgré cette tendance générale, il y a maintenant certaines Africaines qui s'installent au Canada avant leur époux. 